\title{
Synaptic Stimulation of mTOR Is Mediated by Wnt Signaling and Regulation of Glycogen Synthetase Kinase-3
}

\author{
Tao Ma, ${ }^{1}$ Nikos Tzavaras, ${ }^{1}$ Panayiotis Tsokas, ${ }^{1}$ Emmanuel M. Landau, ${ }^{1,2}$ and Robert D. Blitzer ${ }^{1,2}$ \\ Departments of ${ }^{1}$ Pharmacology and Systems Therapeutics and ${ }^{2}$ Psychiatry, Mount Sinai School of Medicine, New York, New York 10029
}

\begin{abstract}
The persistent or "late" phase of long-term potentiation (L-LTP), which requires protein synthesis, can be induced by relatively intense synaptic activity. The ability of such strong synaptic protocols to engage the translational machinery and produce plasticity-related proteins, while weaker protocols activate only posttranslational processes and transient potentiation (early LTP; E-LTP), is not understood. Among the major translation control pathways in neurons, the stimulation of mammalian target of rapamycin (mTOR) is a key event in the induction of L-LTP. We report that mTOR is tonically suppressed in rat hippocampus under resting conditions, a consequence of the basal activity of glycogen synthetase kinase 3 (GSK3). This suppression could be overcome by weak synaptic stimulation in the presence of the $\beta$-adrenergic agonist isoproterenol, a combination that induced L-LTP, and activation of mTOR coincided with the Akt-mediated phosphorylation of GSK3. Surprisingly, while isoproterenol alone elevated Akt activity, it failed to increase GSK3 phosphorylation or mTOR signaling, showing that Akt was uncoupled from these effectors in the absence of synaptic stimulation. With the addition of weak stimulation, Akt signaled to GSK3 and mTOR, a gating effect that was mediated by voltage-dependent $\mathrm{Ca}^{2+}$ channels and the Wnt pathway. mTOR could be stimulated by pharmacological inhibition, enabling weak HFS to induce L-LTP. These results establish GSK3 as an integrator of Akt and Wnt signals and suggest that overcoming GSK3-mediated suppression of mTOR is a key event in the induction of L-LTP by synaptic activity.
\end{abstract}

\section{Introduction}

Following repetitive stimulation of excitatory synapses in the hippocampus, two distinct forms of LTP can be distinguished: the relatively transient E-LTP, which requires only a weak induction protocol and is mediated by an entirely posttranslational mechanism, and the more stable L-LTP, which requires a strong induction protocol and newly synthesized proteins (Frey and Morris, 1997). A detailed understanding of the signaling events that are uniquely evoked by protocols that induce L-LTP should provide insights into the mechanism for long-term memory (LTM) formation, which like L-LTP is persistent, and requires a strong initiating event and protein synthesis.

The protein kinase mammalian target of rapamycin (mTOR) is stimulated by strong and not weak synaptic stimulation, and mTOR activity (specifically, the mTORC1 complex) is required for the induction of L-LTP and formation of LTM (Tsokas et al., 2005; Gafford et al., 2011; Stoica et al., 2011). mTOR generally

Received Sept. 18, 2011; accepted 0ct. 20, 2011.

Author contributions: T.M., P.T., E.M.L., and R.D.B. designed research; T.M., N.T., and P.T. performed research; T.M., N.T., P.T., and R.D.B. analyzed data; T.M., E.M.L., and R.D.B. wrote the paper.

This work was supported by NIH Grants DA029298 (to R.D.B.) and DA015863 (to E.M.L.). We thank Dr. Simon Hardy for his constructive comments on an earlier version of this paper.

Correspondence should be addressed to Robert D. Blitzer, Department of Pharmacology and Systems Therapeutics, Mount Sinai School of Medicine, One Gustave L. Levy Place, New York, NY 10029. E-mail: robert. blitzer@mssm.edu.

T. Ma's present address: Center for Neural Science, New York University, 4 Washington Place, New York, NY 10003.

P. Tsokas's present address: Department of Physiology and Pharmacology, SUNY Downstate Medical Center, 450 Clarkson Avenue, Brooklyn, NY 11203.

DOI:10.1523/JNEUROSCI.4761-11.2011

Copyright $\odot 2011$ the authors $\quad 0270-6474 / 11 / 3117537-10 \$ 15.00 / 0$ serves to match the rate of translation to demands for new proteins that are imposed by extrinsic and intrinsic stimuli. Several aspects of translation are controlled by mTOR, including the synthesis of translational machinery that is encoded by mRNAs with polypyrimidine sequences in their $5^{\prime}$ untranslated regions (TOP mRNAs) (Meyuhas and Hornstein, 2000). TOP mRNAs encode ribosomal proteins and translation elongation factors, some of which are synthesized rapidly from transcripts in dendrites, where an increase in translational capacity may contribute to the localized synthesis of plasticity-related proteins (Tsokas et al., 2007). Following L-LTP induction by highfrequency stimulation (HFS), mTOR activity increases rapidly in dendrites (Tsokas et al., 2005). However, the signaling network that links synaptic stimulation to mTOR activation is largely unknown.

mTOR is regulated by a canonical pathway that includes phosphatidylinositol-3-kinase (PI3K), phosphoinositide-dependent protein kinase 1 (PDK1), and Akt. Additional inputs to mTOR originate from other signaling proteins that have been implicated in L-LTP, most notably extracellular signal-regulated kinase (ERK), which can be activated by neuromodulatory inputs that facilitate L-LTP induction in the hippocampus and are required for LTM (Decker and McGaugh, 1991; O'Dell et al., 2010; Tully and Bolshakov, 2010). Importantly, the contribution of these modulators to L-LTP may be obscured by the use of strong HFS protocols, but can be observed in slices that are stimulated with weak HFS, which becomes capable of inducing L-LTP when combined with a neuromodulator such as a $\beta$-adrenoceptor agonist. In principle, it should be possible to identify the signature signaling events of a strong stimulus by comparing the consequences of 
weak HFS when delivered in the presence versus the absence of such an agonist.

Here, we show that GSK3 plays a central role in the synaptically induced stimulation of mTOR. Basal GSK3 activity suppressed mTOR under resting conditions, an inhibition that was relieved by weak HFS in the presence of isoproterenol, but not by weak HFS alone. The ability of strong stimulation to inhibit GSK3 depended on the convergent effects of Akt and the Wnt pathway, revealing a previously unrecognized level of complexity in the regulation of mTOR during synaptic plasticity.

\section{Materials and Methods}

Hippocampal slice preparation and electrophysiology. Male Sprague Dawley rats $(6-8$ weeks old $)$ were deeply anesthetized with halothane and decapitated. The brain was rapidly removed and chilled in ice-cold artificial CSF (ACSF) containing (in mM) $118 \mathrm{NaCl}, 3.5 \mathrm{KCl}, 2.5 \mathrm{CaCl}_{2}, 1.3$ $\mathrm{MgSO}_{4}, 1.25 \mathrm{NaH}_{2} \mathrm{PO}_{4}, 24 \mathrm{NaHCO}_{3}$, and 15 glucose, bubbled with $95 \%$ $\mathrm{O}_{2} / 5 \% \mathrm{CO}_{2}$. Transverse slices of dorsal hippocampus (450 $\mu \mathrm{m}$ thick) were made on a tissue chopper at $4^{\circ} \mathrm{C}$, and then placed in an interface chamber (ACSF and humidified 95\% $\mathrm{O}_{2} / 5 \% \mathrm{CO}_{2}$ atmosphere), where they were maintained at room temperature for at least $2 \mathrm{~h}$. For recording, slices were transferred to a submersion chamber and superfused with ACSF at $31 \pm 1^{\circ} \mathrm{C}$. Monophasic, constant-current stimuli $(100 \mu \mathrm{s})$ were delivered with a bipolar stainless steel electrode positioned in stratum radiatum of area CA3, and field EPSPs (fEPSPs) were recorded in stratum radiatum of area CA1, using electrodes filled with ACSF $(\operatorname{Re}=2-4 \mathrm{M} \Omega)$. For all slices, initial spike threshold exceeded $2 \mathrm{mV}$. Signals were low-pass filtered at $3 \mathrm{kHz}$ and digitized at $20 \mathrm{kHz}$, and analyzed using Axobasic or pClamp 9 (Molecular Devices). Three HFS protocols were used: Weak HFS, consisting of two 1-s-long $100 \mathrm{~Hz}$ trains, separated by $20 \mathrm{~s}$, and delivered at a stimulus strength that evoked a fEPSP measuring $20 \%$ of spike threshold; Strong HFS, identical to weak HFS but delivered at an intensity that initially evoked a fEPSP of $75-80 \%$ of spike threshold; and ISO-wHFS, in which isoproterenol $(1 \mu \mathrm{M})$ was bath-applied for $10 \mathrm{~min}$, terminating with the delivery of weak HFS. In all experiments, the stimulation protocol was delivered at least $30 \mathrm{~min}$ after transfer of the slices to the recording chamber, when the basal fEPSP had been stable for at least $20 \mathrm{~min}$. Control slices were placed in the recording chamber and subjected only to test stimuli $(0.033 \mathrm{~Hz})$. Drug preincubations, where used, were performed at room temperature in submersion maintenance chambers containing ACSF saturated with bubbling $95 \% \mathrm{O}_{2} / 5 \% \mathrm{CO}_{2}$. Drugs were prepared as stock solutions and diluted to final concentrations in ACSF before use.

Western immunoblotting. Slices were removed from the recording chamber or submersion maintenance chamber and immediately frozen on slides resting on dry ice. The CA1 region was isolated in a cold room $\left(4^{\circ} \mathrm{C}\right)$ under a dissection microscope, and samples were stored at $-80^{\circ} \mathrm{C}$ for $\leq 3 \mathrm{~d}$ before assaying. Each CA1 region was homogenized in $80 \mu \mathrm{l}$ of ice-cold lysis buffer using a motorized Potter-Elvehjem homogenizer directly in the Eppendorf tube ( $8-10$ strokes, 1 stroke per second). The components of the lysis buffer were (in mM, unless indicated otherwise): 25 Tris- $\mathrm{HCl}, \mathrm{pH} 7.4,150 \mathrm{NaCl}, 6 \mathrm{MgCl}_{2}$, 2 EDTA, 1.25\% NP40, 0.125\% SDS, $0.625 \% \mathrm{Na}$ deoxycholate, 4 -nitrophenyl phosphate, $25 \mathrm{Na}$ fluoride, $2 \mathrm{Na}$ pyrophosphate, 20 dithiothreitol, $10 \beta$-glycerophosphate, 1 $\mu \mathrm{M}$ okadaic acid, phosphatase inhibitor cocktail I and II (Calbiochem), 1 phenylmethylsulfonyl fluoride (PMSF), $20 \mu \mathrm{g} / \mathrm{ml}$ leupeptin, and $4 \mu \mathrm{g} / \mathrm{ml}$ aprotinin. Protein determination was performed using Bio-Rad RC-DC Protein Assay kit (Bio-Rad). Appropriate volumes of $4 \times$ NuPage LDS Sample Buffer (Invitrogen) and $\beta$-mercaptoethanol were added to the homogenates, and samples were boiled for $5 \mathrm{~min}$. Samples including appropriate amount of protein were loaded on $8-10 \%$ SDS-PAGE gels and resolved by standard electrophoresis, and proteins transferred electrophoretically onto nitrocellulose membranes $(0.2 \mu \mathrm{m}$ pore size) at $4^{\circ} \mathrm{C}$. Membranes were blocked for at least $30 \mathrm{~min}$ at room temperature with blocking buffer [BB; $5 \%$ nonfat dry milk in TBS containing $0.1 \%$ Tween 20 (TBS-T) ], or with Odyssey blocking buffer (Licor Biosciences), then probed overnight at $4^{\circ} \mathrm{C}$ using primary antibodies for phospho(T389)-p70S6K (1:5000; Cell Signaling Technology),
eEF1A (1:10,000; Millipore), phospho(Thr202/Tyr204)-ERK (1:2000; Cell Signaling Technology), phospho(T308)-Akt (1:1000; Millipore), phospho(S473)-Akt (1:1000, Cell Signaling Technology), phospho(S21/ 9)-GSK3- $\alpha / \beta$ (1:1000, Cell Signaling Technology) phospho(T607)Kv4.2 (1:300, Santa Cruz Biotechnology), Kv4.2 (1:500, Millipore), phospho(T246)-PRAS40 (1:1000; Millipore), phospho(T172)-AMPK (1:1000, Cell Signaling Technology), phospho(S79)-ACC (1:1000, Cell Signaling Technology), and actin (1:5000; Sigma-Aldrich). All primary antibodies were dissolved in BB, or Odyssey buffer plus $0.01 \%$ SDS and $0.1 \%$ Tween. After washing in TBS-T ( 3 washes, 5 min each), the membranes were incubated with horseradish peroxidase-conjugated antirabbit or anti-mouse IgG (1:5000; Pierce Biotechnology), or with Licor IRDye conjugated secondary antibodies (1:5000). Proteins were visualized by chemiluminescence (GE Healthcare ECL Western Blotting Analysis System) or by infrared direct detection using the Odyssey Classic system (Licor). Densitometric analysis of the bands was performed using Scion Image software (Scion Corporation) for ECL, or with Odyssey v.3.0 for direct IR. Data were analyzed using one-tailed and (where specified) two-tailed $t$ tests or, where appropriate, ANOVAs followed by Tukey post hoc tests, using Prism (GraphPad Software) and Origin (OriginLab). Summary data are presented as group means \pm SEMs.

Immunohistochemistry and confocal microscopy. Slices were fixed overnight in ice-cold $4 \%$ paraformaldehyde $/ 0.1 \%$ glutaraldehyde in PBS, $\mathrm{pH}$ 7.4, then washed with PBS before cutting $40 \mu \mathrm{m}$ thick sections on a vibratome (Leica VT 1000S). Free-floating sections were blocked with $10 \%$ normal goat serum, $1 \% \mathrm{BSA}$, and $0.1 \% \mathrm{Na}$ azide in PBS for $2 \mathrm{~h}$, and incubated overnight at $4^{\circ} \mathrm{C}$ with primary antibodies in $1 \%$ BSA. The following primary antibodies were used: phospho(Thr389)-p70S6K (1: 250; Cell Signaling Technology), phospho-GSK3- $\alpha$ (1:250; Cell Signaling Technology), phospho-GSK3- $\beta$ (1:250; Cell Signaling Technology), and $\beta$-tubulin (1:500; Sigma) as a control for antibody penetration. After washing in PBS, sections were incubated in $1 \%$ BSA with secondary antibodies complexed to either Alexa Fluor 568 or Alexa Fluor 488 (both used at 1:250; Invitrogen). For experiments using serial immunohistochemistry, slices were washed extensively after the first secondary incubation before applying the next primary antibody. Processed sections were mounted and imaged confocally (Zeiss LSM meta-510) at a scanning depth of $1.9 \mu \mathrm{m}(200 \times)$ or $0.9 \mu \mathrm{m}(400 \times)$. Alexa Fluor $488 \mathrm{immu}-$ nofluorescence was detected with an ArKr 488/568 laser set at 505-545 $\mathrm{nm}$ bandpass emission. The same laser was used with a $560-615 \mathrm{~nm}$ long-pass emission filter for visualizing Alexa Fluor 568. All parameters (pinhole, contrast, and brightness) were held constant for all sections from the same experiment. For double-label experiments, scans at different wavelengths were digitally merged.

Drugs. All drugs were prepared as stock solutions in water or DMSO and diluted to final concentrations in ACSF before use. The following drugs, final concentrations, and sources were used: ( - -)-isoproterenol (+)-bitartrate ( $1 \mu \mathrm{M}$; Sigma), emetine ( $20 \mu \mathrm{M}$; Calbiochem), rapamycin

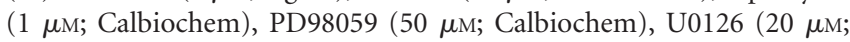
Calbiochem), D-APV (50 $\mu \mathrm{M}$; Calbiochem), nifedipine (10 $\mu \mathrm{M}$; Calbiochem), kenpaullone (1 $\mu \mathrm{M}$; Calbiochem), $\mathrm{LiCl}$ (10 mM, Sigma), Akt inhibitor VIII ( $5 \mu \mathrm{M}$; Calbiochem), Frizzled-8/Fc chimera ( $5 \mu \mathrm{g} / \mathrm{ml}$; R\&D Systems), and dorsomorphin (50 $\mu \mathrm{M}$; Calbiochem). When a drug was preapplied in the maintenance chamber, the same drug was present, at the same concentration, in the recording chamber for the duration of the experiment.

\section{Results}

Weak HFS combined with isoproterenol induces mTOR- and ERK-dependent late LTP

Weak HFS (wHFS) delivered to the Schaffer collaterals produced a transient potentiation at CA1 synapses, which decayed to baseline within $3 \mathrm{~h}$ (E-LTP; Fig. $1 A$ ). In contrast, the same stimulation delivered in the presence of the $\beta$-adrenergic agonist isoproterenol (ISO-wHFS protocol) induced a more sustained potentiation (L-LTP) that required protein synthesis, since only transient potentiation remained in slices treated with the translation inhibitor emetine (Fig. $1 A, B$ ). The induction of L-LTP by 

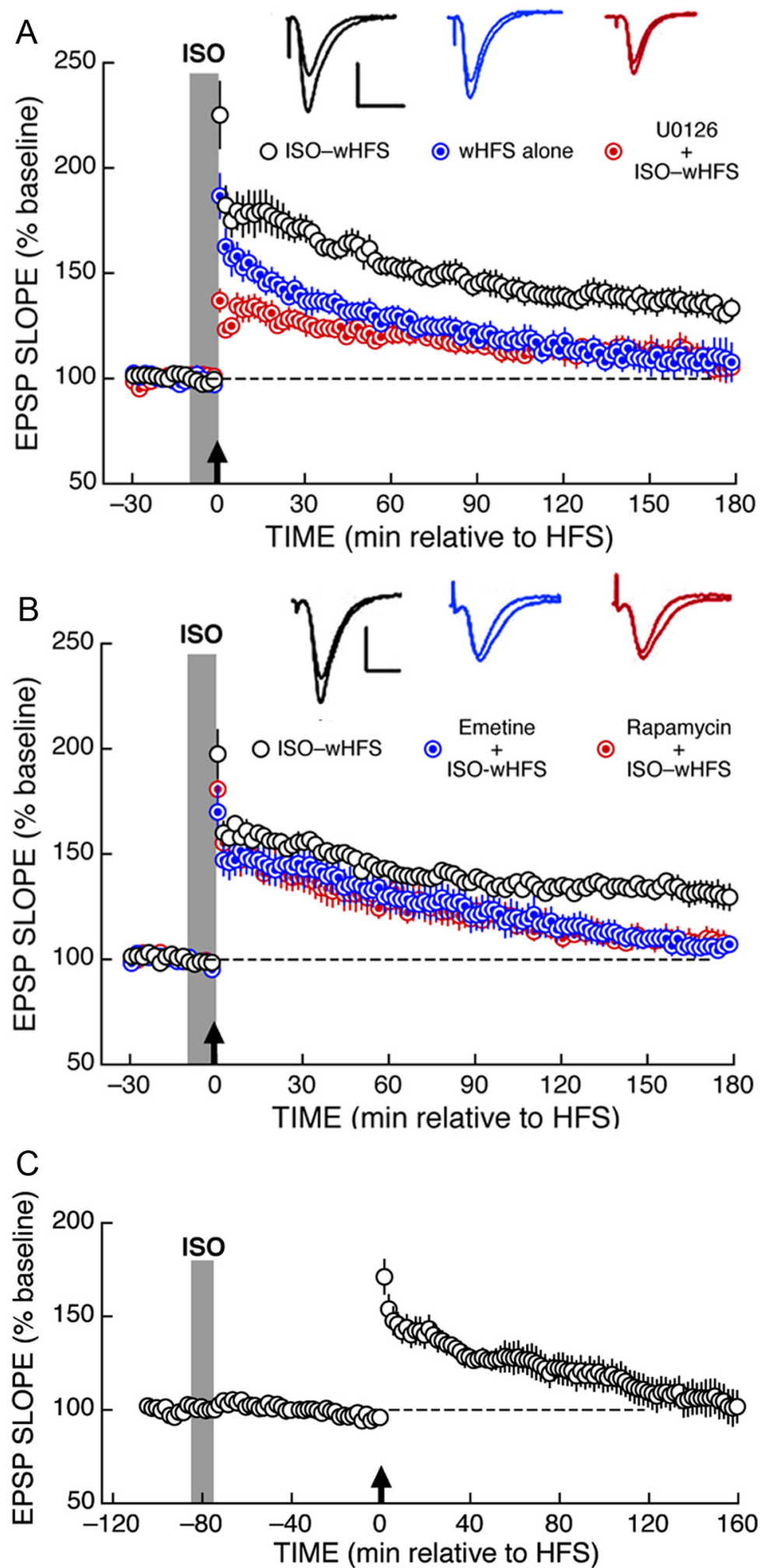

Figure 1. Weak HFS delivered in the presence of isoproterenol induces ERK-and mTOR-dependent late LTP. $A$, Pairing weak HFS with $1 \mu \mathrm{m}$ isoproterenol (ISO-wHFS) induced L-LTP (open circles, $n=14$ ), whereas weak HFS alone induced only a transient E-LTP (blue circles, $n=14$ ). ISO-wHFS failed to induce LTP in slices treated with the ERK pathway inhibitor U0126 (20 $\mu \mathrm{m}$; red circles, $n=$ 4). Calibration: $0.5 \mathrm{mV}, 20 \mathrm{~ms}$. The sample traces, here and in $\boldsymbol{B}$, were taken $5 \mathrm{~min}$ before and $180 \mathrm{~min}$ after HFS. $\boldsymbol{B}$, The induction of L-LTP by ISO-wHFS (open circles, $n=6$ ) was blocked by pretreatment with emetine ( $20 \mu \mathrm{m}$; blue circles, $n=4)$ or rapamycin $(1 \mu \mathrm{m}$; red circles, $n=4)$. Calibration: $0.5 \mathrm{mV}, 10 \mathrm{~ms}$. C, Weak HFS that was delivered $75 \mathrm{~min}$ after isoproterenol washout induced only E-LTP.
ISO-wHFS also depended on ERK activity, since slices that were pretreated with the MEK inhibitor U0126 (Fig. 1A) or the structurally unrelated PD98059 (data not shown) expressed only E-LTP, consistent with previous results obtained with ISOwHFS in mouse hippocampus (Gelinas et al., 2007). ISO-wHFS did not induce L-LTP in slices that were pretreated with the specific mTOR inhibitor rapamycin (Fig. 1B). Isoproterenol by itself had no effect on synaptic efficiency, and weak HFS failed to induce L-LTP if it was delayed until $75 \mathrm{~min}$ after isoproterenol (Fig. 1C). Therefore, concurrent $\beta$-adrenergic stimulation and weak HFS contributed synergistically to the induction of L-LTP.

The coupling of Akt to mTOR is conditional on synaptic stimulation To gain insights into the signaling events that lead to L-LTP, we studied pathways that might underlie the synergistic relationship between isoproterenol and weak HFS, focusing on mTOR regulation in area CA1. We previously showed that our weak HFS protocol does not, by itself, stimulate mTOR (Tsokas et al., 2005). However, $\beta$-adrenergic stimulation activates ERK, which is an early noncanonical component of the mTOR regulatory network, indirectly stimulating PDK1 (Frödin et al., 2000; Gelinas et al., 2007; Tsokas et al., 2007). Thus, we tested whether isoproterenol increases mTOR signaling, and in this way enables the E-LTP that is induced by weak HFS to proceed to L-LTP.

Isoproterenol by itself activated ERK in area CA1, as indicated by the phosphorylation of ERK at T202/Y204 (Fig. 2A). More importantly, isoproterenol also induced the phosphorylation of Akt at the PDK1-dependent site T308, in an ERKdependent manner (Fig. 2B). Weak HFS alone did not increase ERK or Akt phosphorylation, and the effects of ISO-wHFS on these measures were no greater than those of isoproterenol alone (Fig. $2 A, B$ ). Thus, the activation of the mTOR regulatory pathway by ISO-wHFS, at least as far downstream as Akt, was mediated entirely by $\beta$-adrenergic activation with no contribution from weak HFS.

Unexpectedly, further downstream, the stimulatory effect of isoproterenol did not extend to mTOR itself. mTOR activity was monitored by phosphorylation of p70-S6 kinase (p70S6K) at its mTOR-dependent site T389, and by the expression of a TOP mRNA-encoded protein, translation elongation factor 1A (eEF1A). Neither isoproterenol nor 

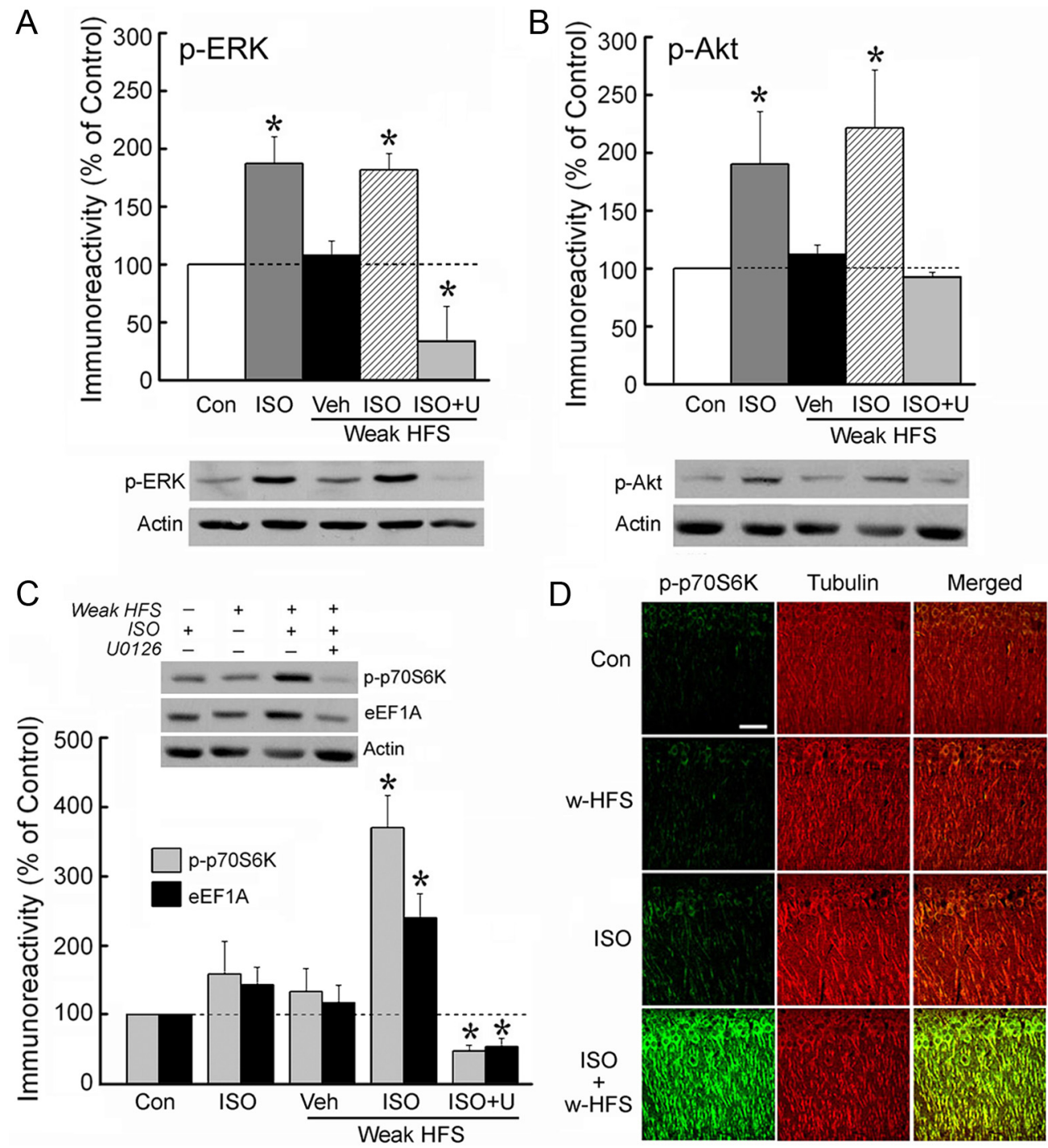

Figure 2. The coupling of Akt to mTOR is conditional and enabled by weak HFS in the presence of isoproterenol. $A$, Isoproterenol alone (ISO), but not weak HFS alone, increased the phosphorylation of ERK at T202/Y204 in area CA1. A similar level of ERK phosphorylation was produced by the combined ISO-wHFS protocol (compare lanes 2 and 4). All group $n$ values $\geq 6$. The asterisks, here and unless otherwise noted, indicate significant differences $(p<0.05)$ from control slices that received only test stimuli. $\boldsymbol{B}$, Isoproterenol alone increased Akt phosphorylation at T308 to the same extent as ISO-wHFS (compare lanes 2 and 4), while weak HFS alone had no effect. The phosphorylation of Akt induced by ISO-wHFS was completely blocked by U0126 (20 $\mu$ M). All group $n$ values $\geq 6$. C, Isoproterenol alone did not increase p7056K phosphorylation (at T389) or eEF1A expression above their basal levels ( $p$ values $>0.05$ ). However, both measures were significantly increased by ISO-wHFS, and these effects were prevented by incubation with U0126 $(20 \mu \mathrm{m})$. All group $n$ values $\geq 8$. D, ISO-wHFS increased immunoreactivity for phospho-p70S6K in the apical dendrites and cell bodies of CA1 pyramidal cells. Images are representative of three experiments, with little or no effect of isoproterenol or weak HFS alone. Scale bar, $100 \mu \mathrm{m}$.

weak HFS alone increased p70S6K phosphorylation or eEF1A expression above baseline levels (Fig. 2C). However, the combined ISO-wHFS protocol produced robust increases in both measures, indicating that signaling between Akt and mTOR was contingent on a factor that is produced by synaptic stimulation in the presence of isoproterenol. When ERK was inhibited, ISO-wHFS failed to stimulate mTOR (Fig. 2C), and in fact reduced p70S6K phosphorylation and eEF1A expression below control levels, suggesting that ISO-wHFS may activate proteases and protein phosphatases whose effects are revealed when ERK is inhibited. The spatial distribution of increased mTOR signaling included the apical dendrites and cell bodies of CA1 pyramidal neurons (Fig. $2 D$ ), similar to previous studies where strong HFS was used to induce L-LTP (Cammalleri et al., 2003; Tsokas et al., 2005).

\section{$\mathrm{Ca}^{2+}$ channels mediate the conditional coupling of Akt} to $\mathrm{mTOR}$

To identify the signaling events that enable signal propagation from Akt to mTOR, we sought ERK-dependent effects that are reliably produced by ISO-wHFS, but not by weak HFS or isoproterenol alone. One prominent role of ERK in synaptic plasticity is based on its ability to phosphorylate and inhibit Kv4.2 potassium channels, an effect that increases dendritic excitability and probably allows $\mathrm{Ca}^{2+}$ influx during dendritic spikes that are evoked during repeated excitatory stimulation (Yuan et al., 2002; Cai et al., 2004; Schrader et al., 2006). We found that ISO-wHFS produced an ERK-dependent phosphorylation of Kv4.2 at the inhibitory site T607. This effect was due entirely to isoproterenol treatment, with no additional effect of concurrent weak HFS (Fig. 3A).

$\mathrm{Ca}^{2+}$ influx during dendritic spiking might play a role in mTOR signaling, since $\mathrm{Ca}^{2+}$ entry has been shown to increase 

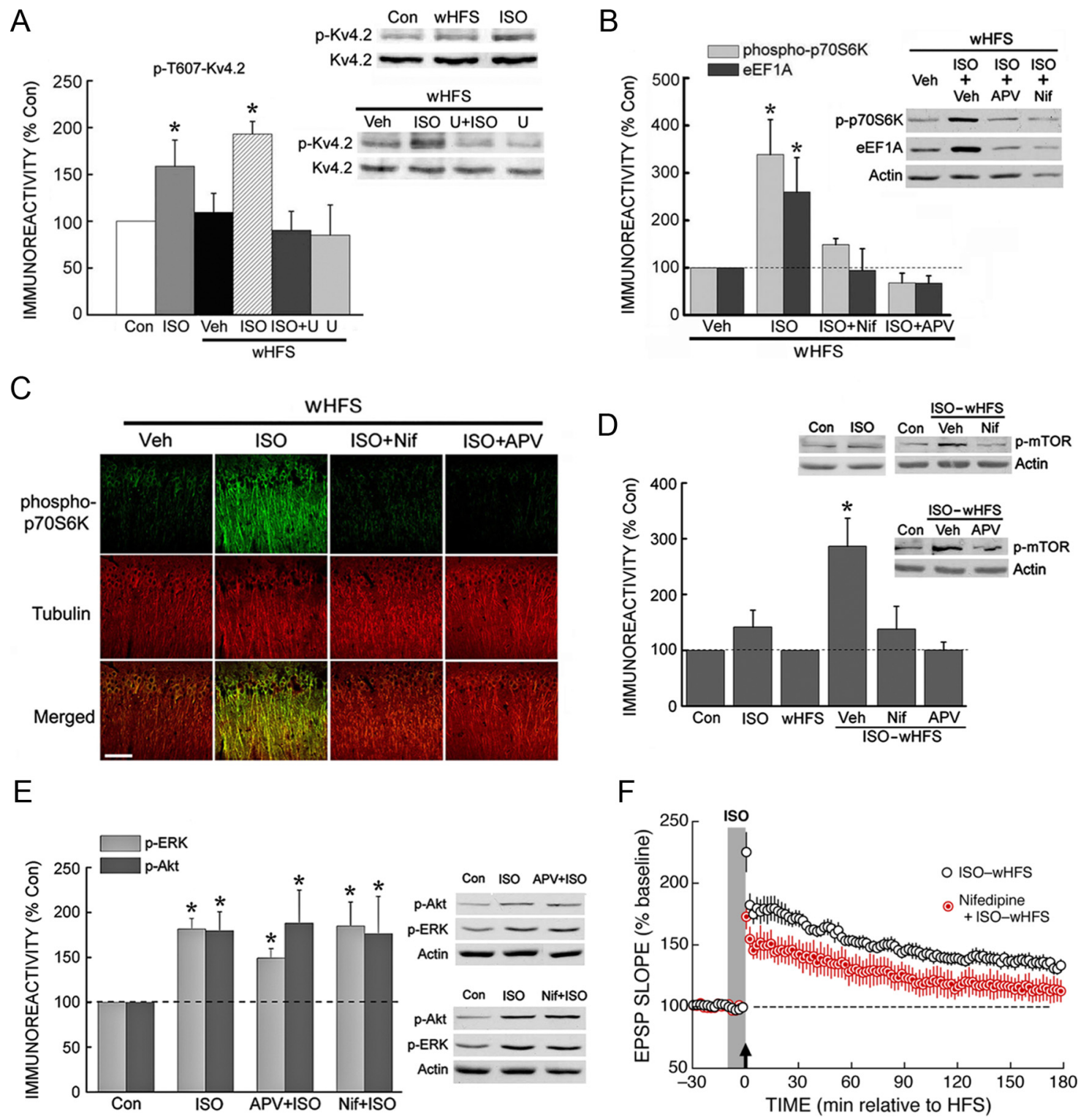

Figure 3. The coupling of Akt to mTOR is mediated by L-type VGCCs and NMDARs. $A$, Isoproterenol (1 $\mu \mathrm{M})$ alone induced ERK-dependent hyperphosphorylation of Kv4.2 channels at T607, with no additional effect of concurrently delivered weak HFS. All $n$ values $=6 . \boldsymbol{B}$, In slices treated with nifedipine (Nif; $10 \mu \mathrm{M}$ ) or D-APV (50 $\mu \mathrm{M}$ ), ISO-wHFS failed to increase p70S6K phosphorylation or eEF1A expression ( $n=6$ for all groups). Here and in $\boldsymbol{D}$, asterisks indicate $p<0.05$ versus weak HFS-only controls. $\boldsymbol{C}$, Both the dendritic and somatic increases in phospho-p7056K following ISO-wHFS were blocked when stimulation was delivered in the presence of nifedipine or D-APV. The images are representative of three independent experiments. Scale bar, $100 \mu \mathrm{m}$. D, Nifedipine and D-APV also prevented the ISO-wHFS-induced phosphorylation of mTOR at S2481, an autophosphorylation site. All group $n$ values $\geq 5$. $E$, Neither nifedipine nor D-APV reduced the ability of isoproterenol to increase the phosphorylation level of ERK or Akt. All $n$ values $>5 . F$, Incubation of slices with nifedipine interfered with the induction E-LTP [as defined by insensitivity to emetine (Fig. $1 B$ )] and L-LTP by ISO-wHFS. Both group $n$ values $\geq 5$.

mTOR activity (Tang et al., 2003; Lenz and Avruch, 2005; Kwon et al., 2006). This influx is carried, in part, by L-type voltage-gated $\mathrm{Ca}^{2+}$ channels (VGCCs), whose contribution is increased by $\beta$-adrenergic receptor activation (Hoogland and Saggau, 2004). When L-type VGCCs were blocked by nifedipine, ISO-wHFS failed to increase p70S6K phosphorylation or eEF1A expression, and inhibition of NMDA-type glutamate receptor (NMDARs) by D-APV also prevented these increases (Fig. $3 B, C$ ). Similarly, L-type VGCCs and NMDARs were required for the ISO-wHFSinduced phosphorylation of mTOR at its autophosphorylation site S2481 (Brown et al., 1995; Peterson et al., 2000) (Fig. 3D). Importantly, neither nifedipine nor D-APV prevented isoproterenol from stimulating ERK or Akt (Fig. 3E). Thus, the activation of $\mathrm{Ca}^{2+}$-permeable channels during ISO-wHFS serves to gate signaling downstream of Akt, and either upstream of mTOR or at mTOR itself.

Since L-type VGCCs are required for mTOR stimulation by ISO-wHFS, the induction of L-LTP by this induction protocol also should depend on these channels. In agreement, L-LTP was significantly reduced in slices that were incubated with nifedipine (Fig. 3F).

\section{Phosphorylation of GSK3 $\alpha$ mediates the $\mathrm{Ca}^{2+}$-dependent coupling of Akt to mTOR}

The mechanism for the conditional coupling of Akt to mTOR must involve signaling molecules outside of the canonical pathway, in which Akt activates mTOR by inhibiting the mTOR suppressor TSC2. We tested a series of non-canonical regulators of 

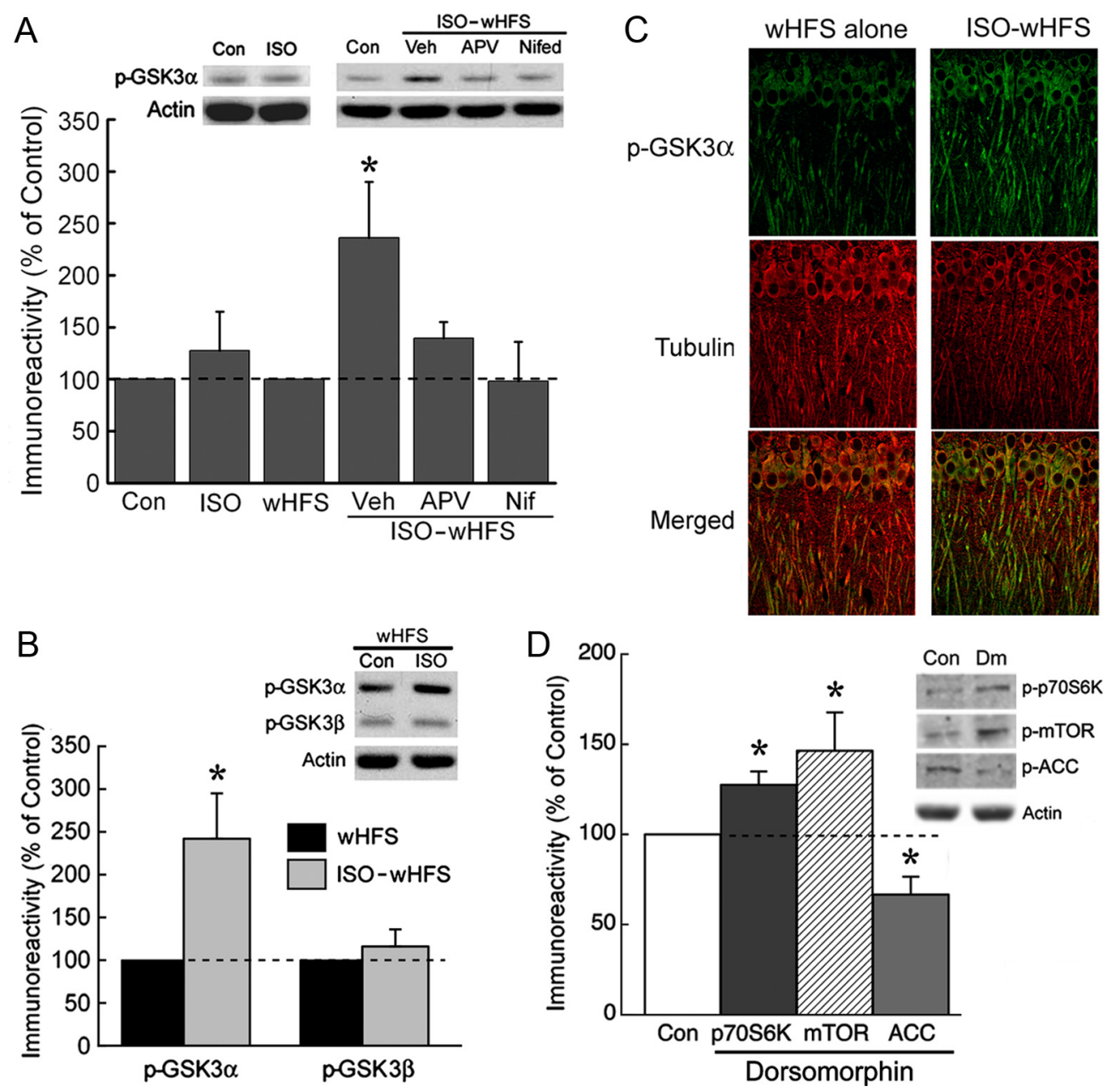

Figure 4. Increases in mTOR signaling correlate with the inhibitory phosphorylation of GSK3 $\alpha$. A, ISO-wHFS significantly enhanced the phosphorylation of GSK3 $\alpha$ at $S 21$ ( $p<0.05$ vs controls), and this effect was completely blocked by D-APV $(50 \mu \mathrm{m})$ and nifedipine $(10 \mu \mathrm{M})$. In contrast, treatment with isoproterenol or weak HFS alone did not affect GSK3 $\alpha$ phosphorylation. $n=6$ for all groups. $\boldsymbol{B}$, In slices treated with ISO-wHFS, GSK3 phosphorylation was increased in the somatic and apical dendritic regions in area CA1. The images are representative of three experiments. Scale bar, $50 \mu \mathrm{m}$. C, The ability of ISO-wHFS to induce GSK3 phosphorylation was specific to the $\alpha$ isoform ( $p<0.05$ vs weak HFS only control), with no effect on GSK3 $\beta$. Both group $n$ values $\geq 5$. Asterisk indicates significant difference from weak HFS-only control $(p<0.05)$. $\boldsymbol{D}$, Treatment of slices with the AMPK inhibitor dorsomorphin (Dm) increased the phosphorylation levels of p70S6K (at T389) and mTOR (at S2481), while decreasing phosphorylation of the AMPK substrate acetyl-CoA carboxylase (ACC) at S79. Group $n$ values $\geq 9$.

mTOR signaling, several of which proved to be unaffected by ISO-wHFS and thus not potential participants in the coupling process. These mechanisms included phosphorylation of Akt at S473, which increases catalytic activity above that produced by T308 phosphorylation alone (Manning and Cantley, 2007), and Akt-mediated phosphorylation of the mTOR suppressors PRAS40 (proline-rich Akt/PKB substrate $40 \mathrm{kDa}$ ) and AMPK (AMP-activated protein kinase) at their inhibitory sites T246 and T172, respectively (Hahn-Windgassen et al., 2005; Sancak et al., 2007; Vander Haar et al., 2007; Wang et al., 2007) (data not shown).

mTOR also can be regulated by glycogen synthetase 3 (GSK3), an enzyme that is active in its basal state and inhibits mTOR by stimulating TSC2 (Inoki et al., 2006). In vitro, GSK3 is inhibited by protein kinases that are relevant to synaptic plasticity in the hippocampus, including Akt, p70S6K, and RSK (Srivastava and Pandey, 1998). We found that ISO-wHFS significantly increased the phosphorylation of the $\alpha$-isoform of GSK3 at its Aktdependent site, S21 while isoproterenol or weak HFS applied individually had no effect (Fig. 4A). The effect of ISO-wHFS on GSK3 $\alpha$ phosphorylation was fully blocked by nifedipine and by D-APV, indicating that the inhibition of GSK3 by Akt depends on
$\mathrm{Ca}^{2+}$ conductances that mediate L-LTP induction by this protocol (Fig. 4A). Furthermore, ISO-wHFS increased GSK3 $\alpha$ phosphorylation throughout the somatic and apical dendritic regions in area CA1 (Fig. 4C). Surprisingly, ISO-wHFS did not increase phosphorylation of the more commonly studied $\beta$-isoform of GSK3 at S9 (the corresponding Akt-dependent site) (Fig. 4B). In non-neuronal cells, mTOR activation is dependent on AMPK, which is required to prime TSC2 for subsequent phosphorylation by GSK3 (Inoki et al., 2006), and in agreement with this mechanism, we found that the pharmacological inhibition of AMPK stimulated mTOR signaling (Fig. $4 D$ ). Note that basal AMPK activity apparently sufficed to prime TSC2 under our conditions, since ISO-wHFS did not affect the phosphorylation state of AMPK at its regulatory site T172, as described above.

If the ISO-wHFS-induced inhibition of GSK3 is responsible for coupling Akt to mTOR in the hippocampus, then the high basal activity of GSK3 might act as a brake on mTOR signaling under resting conditions; if so, it should be possible to stimulate mTOR by pharmacologically inhibiting GSK3. This prediction was confirmed by using two structurally unrelated GSK3 inhibitors, $\mathrm{LiCl}$ and kenpaullone, which increased p70S6K phosphorylation and eEF1A expression in otherwise unstimulated slices 

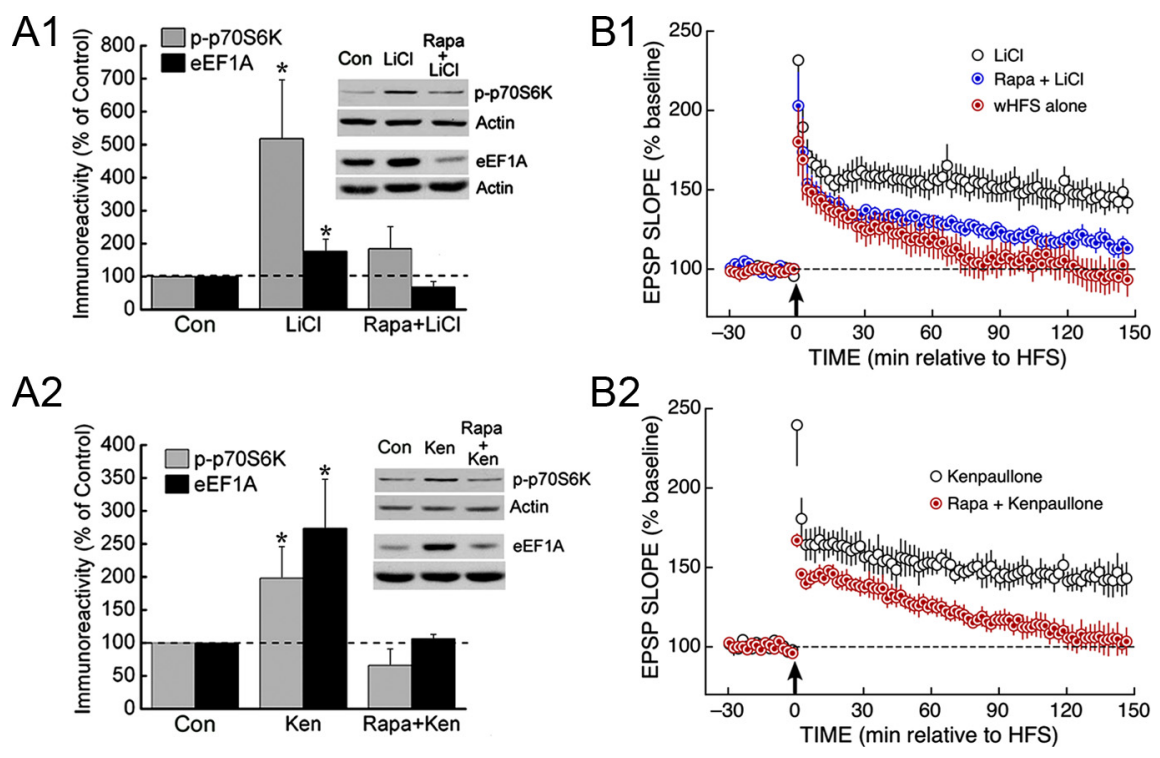

Figure 5. Inhibition of GSK3 upregulates mTOR signaling and enables weak HFS to induce persistent LTP. $A$, The GSK3 inhibitors $\mathrm{LiCl}(10 \mathrm{~mm} ; \boldsymbol{A 1})$ or kenpaullone (1 $\mu \mathrm{m} ; A 2)$ increased the levels of p7056K phosphorylation (at T389) and eEF1A expression. These effects were prevented by pretreatment with rapamycin $(1 \mu \mathrm{M})$. All $n$ values $\geq 5$. $\boldsymbol{B}$, In the presence of LiCl $(10 \mathrm{~mm} ; \boldsymbol{B} \mathbf{1})$ or kenpaullone (1 $\mu \mathrm{M} ; \boldsymbol{B} 2$ ), weak HFS induced L-LTP that was sensitive to $1 \mu \mathrm{m}$ rapamycin. All group $n$ values $\geq 4$.

activation of the Wnt pathway (Chen et al., 2006) might give Akt access to GSK3. We tested this hypothesis by inhibiting Wnt signaling with the scavenger Frizzled-8-Fc. In the presence of the scavenger, ISO-wHFS failed to induce phosphorylation of GSK3, and $\mathrm{mTOR}$ activity was not increased above baseline, as indicated by $\mathrm{p} 70 \mathrm{~S} 6 \mathrm{~K}$ phosphorylation (Fig. 6B). Moreover, pretreatment of slices with Frizzled-8-Fc blocked the induction of L-LTP by ISO-wHFS (Fig. 6C). Since the only known effect of Wnt signaling on GSK3 involves the regulation of the destruction complex, it is likely that the conditional ability of Akt to phosphorylate GSK3 depends on the activation of Wnt signaling. The ability of Frizzled-8-Fc to eliminate the increase in GSK3 phosphorylation shows that Akt had access only to the pool of GSK3 that is under control of Wnt. As expected from our observation that Akt mediates the inhibitory phosphorylation of GSK3, ISOwHFS failed to induce L-LTP when Akt was inhibited (Fig. 6C).

Since the Wnt-mediated loss of GSK3

(Fig. 5A). These effects were mediated by mTOR, since they were abolished by treatment with rapamycin (Fig. 5A).

We next asked whether direct inhibition of GSK3 could substitute for $\beta$-adrenergic stimulation and enable weak HFS to induce L-LTP, as expected if the suppression of mTOR by basal GSK3 activity is critical for regulating the progression from E-LTP to L-LTP. When weak HFS was delivered in the presence of $\mathrm{LiCl}$ or kenpaullone, it produced rapamycin-sensitive L-LTP (Fig. 5B). Together, our findings indicate that $\mathrm{Ca}^{2+}$-dependent inhibition of GSK3 plays a key role in activating the mTOR pathway in the hippocampus, thereby facilitating the induction of L-LTP by synaptic stimulation.

\section{Wnt signaling is required for the Akt-mediated phosphorylation of GSK $3 \alpha$}

How does the activation of L-type VGCCs and NMDARs lead to the phosphorylation and inhibition of GSK3? We have shown that isoproterenol alone stimulates Akt without a concomitant increase in GSK3 phosphorylation, while ISO-wHFS produces the same degree of Akt stimulation but additionally increases phospho-GSK3 levels (Figs. $2 \mathrm{~B}, 4 \mathrm{~A}$ ). A selective inhibitor of Akt prevented the ISO-wHFS-induced phosphorylation of GSK3 (Fig. 6A), so it is unlikely that some other protein kinase is responsible for the regulation of GSK3 by this stimulation protocol. In addition, the phosphorylation of GSK3 required L-type VGCCs and NMDARs, which were not involved in the activation of Akt (Fig. 4A). Together, these observations suggest that Akt has reduced access to its phosphorylation site on GSK3 under resting conditions, while $\mathrm{Ca}^{2+}$ entry associated with ISO-wHFS makes GSK3 susceptible to phosphorylation by Akt.

Interestingly, GSK3 is a component of the "destruction complex," which is best known as a regulator of $\beta$-catenin levels and is disrupted by activation of the canonical Wnt pathway (Logan and Nusse, 2004). Previous work indicates that this pool of GSK3 regulates mTOR, by maintaining high activity of the mTOR suppressor TSC2 (which also is a component of the destruction complex) (Inoki et al., 2006). If GSK3 in the destruction complex is protected from Akt, then stimulation-evoked exocytosis of Wnt and consequent interaction with $\beta$-catenin leads to the upregulation of Tcf/Lefpromoted genes (MacDonald et al., 2009), it is possible that newly transcribed genes play a role in the ability of kenpaullone to facilitate L-LTP induction by weak HFS. However, pretreatment of slices with the transcription inhibitor actinomycin-D did not reduce the effectiveness of kenpaullone (Fig. $6 D$ ). Therefore, although kenpaullone may have produced $\beta$-catenin-mediated changes in gene expression, these did not contribute to the induction of L-LTP or its expression for the duration of our recordings.

Our results suggest that GSK3 serves to integrate ERK, Akt, and Wnt signals in the induction of L-LTP. If GSK3 plays this role, then the pharmacological inhibition of GSK3 should render L-LTP insensitive to inhibitors of ERK, Akt, and Wnt. To test this prediction, we challenged the ability of weak HFS to induce L-LTP in the presence of kenpaullone (as in Fig. 5B2) with U0126, Akt-I, and Frizzled-8-Fc. Under all of these conditions, L-LTP induction was intact (Fig. 6E), indicating that GSK3 is a downstream effector for the ERK, Akt, and Wnt pathways, and the convergent effects of these inputs on GSK3 allows signal propagation downstream from Akt to mTOR.

\section{Discussion}

The conditional coupling of Akt to $\mathrm{mTOR}$ is a novel regulated event in the induction of L-LTP

A defining feature of the strong synaptic protocols that induce late forms of plasticity is the ability to upregulate protein synthesis, although the signaling network that links synaptic activity to translational effectors is poorly understood. To gain insights into this network, we focused on signaling pathways upstream of the translational effector mTOR. An unexpected outcome of these studies was the conditional coupling of Akt to downstream components of the mTOR pathway: while the activation of $\beta$-adrenergic receptors alone was effective in stimulating Akt, this signal propagated downstream to mTOR only in neurons that received concurrent synaptic stimulation. The regulated coupling of Akt to mTOR represents a novel translation control mechanism associated with the induction of L-LTP, and implies the existence of a gating mechanism that integrates con- 
A
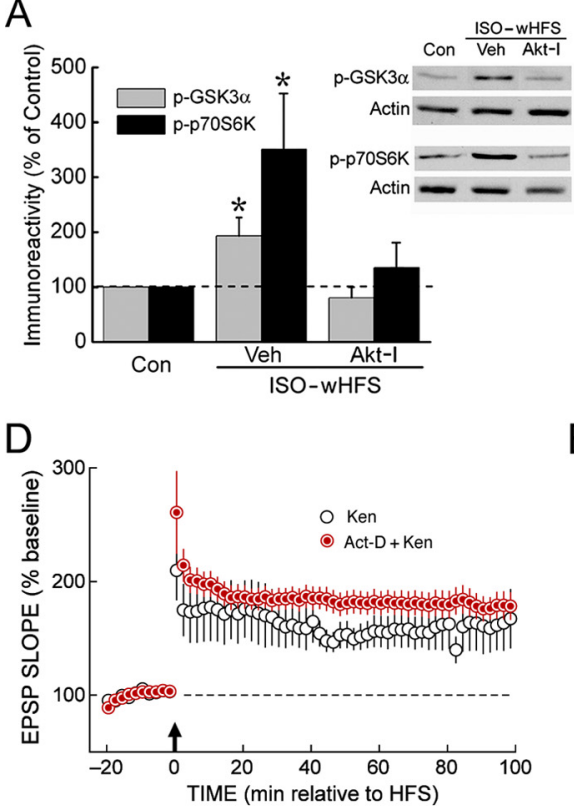

B

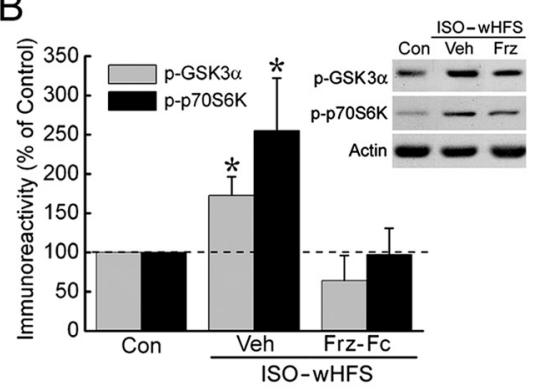

$\mathrm{E}$

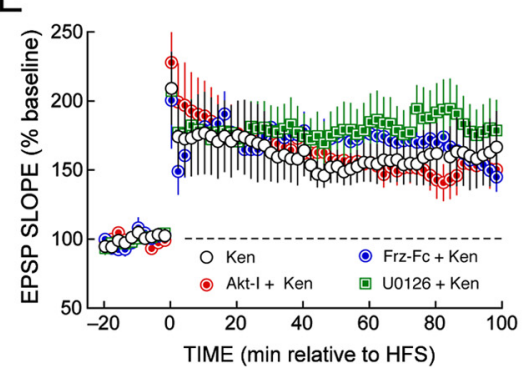

C

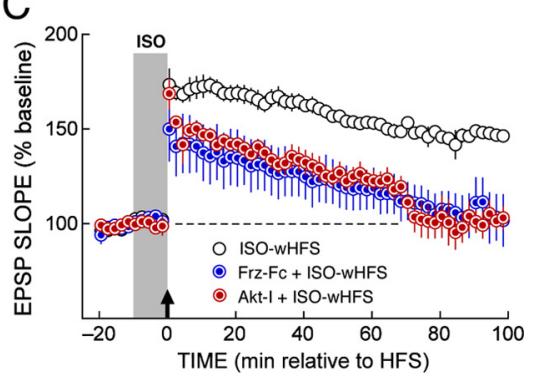

$\mathrm{F}$
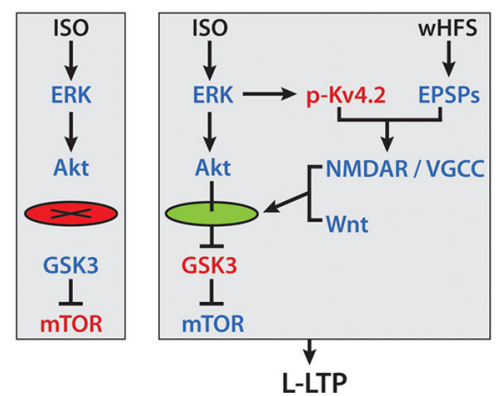

Figure 6. Akt and Wnt mediate the ISO-wHFS-induced phosphorylation of GSK3 $\alpha$ and p70S6K. A, Akt inhibitor VIII, a selective noncompetitive inhibitor of Akt (Akt-l; $5 \mu \mathrm{M}$ ), prevented the phosphorylation of GSK3 $\alpha$ (at S21) and p70S6K (at T389) following ISO-wHFS. Group $n$ values $\geq 4$. B, ISO-wHFS failed to induce phosphorylation of GSK3 $\alpha$ and p70S6K in slices treated with the Wnt scavenger Frizzled-8-Fc ( $F r z-F c ; 5 \mu \mathrm{g} / \mathrm{ml})$. All $n$ values $\geq 4$. C, In slices treated with the Akt inhibitor Akt-I, or with the Wnt antagonist Frizzled-8-Fc, ISO-wHFS failed to induce L-LTP. All group $n$ values $\geq 5$. D. Inhibition of gene transcription with actinomycin-D (Act-D; $40 \mu \mathrm{M}$ ) did not interfere with the induction of L-LTP by weak HFS delivered in the presence of the GSK3 inhibitor kenpaullone (Ken). Group $n$ values $\geq 5$. E, L-LTP induced by wHFS in the presence of kenpaullone was insensitive to inhibitors of ERK (U0126, $20 \mu \mathrm{M}$ ) and Akt (Akt-I, $5 \mu \mathrm{M})$, and to the Wnt antagonist Frizzled-8-Fc $(5 \mu \mathrm{g} / \mathrm{ml})$. All $n$ values $\geq 5$. $F$, In a model for mTOR activation that is based on the results of this study, isoproterenol alone (left panel) stimulates ERK and consequently Akt. However, GSK3 is inaccessible to Akt, and mTOR activity remains in its low resting state. Isoproterenol-induced ERK activity also inhibits Kv4.2 channels through ERK (right panel), increasing dendritic excitability and allowing concurrently delivered weak HFS to recruit dendritic NMDARs and L-type VGCCs. The activation of these $\mathrm{Ca}^{2+}$ channels and signaling through the canonical Wnt pathway serve to relieve the barrier between Akt and GSK3. The consequent inhibitory phosphorylation of GSK3, together with disrupted signaling of GSK3 within the destruction complex (see Discussion), reduces the suppression of $\mathrm{mTOR}$, thereby increasing the translational capacity of the dendrite and enabling the induction of L-LTP. Components shown in blue text are in high-activity states, and those in red text are in low-activity states. The lines that connect components in this network do not necessarily represent direct interactions.

current $\beta$-adrenergic and glutamatergic signals. Subsequent experiments showed that GSK3 inhibition, under the control of Wnt, is critical for this gating process.

In agreement with our results, previous work in mouse hippocampus showed that ISO-wHFS induced L-LTP, while neither isoproterenol nor weak HFS was effective alone (Gelinas et al., 2007). Interestingly, the signaling mechanism that integrated $\beta$-adrenergic stimulation and weak HFS in that study differed from the one described here. We found strongly synergistic effects of concurrent $\beta$-adrenergic and synaptic stimulation on mTOR in the rat hippocampus, while Gelinas et al. (2007) showed additive effects in the mouse; therefore, the cellular decision to commit to L-LTP must be made downstream of mTOR in the mouse hippocampal neurons. Since the experimental protocols used here and in Gelinas et al. (2007) were similar, it is possible that signal propagation from Akt to mTOR is an unregulated process in the mouse. However, the induction of LTP in mouse dentate gyrus depends on exogenous Wnt signaling and can be enhanced by inhibition of GSK3 (Chen et al., 2006; Ma et al., 2010), suggesting that these non-canonical elements of the mTOR regulatory network can play roles in plasticity-related translation in mouse hippocampus.

\section{$\mathrm{Ca}^{2+}$ entry and Wnts mediate synaptically induced} mTOR signaling

We identified L-type VGCCs and NMDARs as critical signaling components for coupling Akt to mTOR. Importantly, the activation of ERK and Akt by ISO-wHFS was unaffected by nifedipine and D-APV, so $\mathrm{Ca}^{2+}$ must act downstream of Akt, enabling signal propagation to mTOR. In contrast to L-LTP induction by ISOwHFS, strong HFS protocols can produce LTP without a contribution from nifedipine-sensitive channels (Teyler et al., 1994), suggesting that $\mathrm{Ca}^{2+}$ influx through NMDARs during intense synaptic activity is sufficient to couple Akt to mTOR. With more moderate induction protocols, such as ISO-wHFS, a cooperative interaction between NMDARs and VGCCs might be essential for $\mathrm{Ca}^{2+}$ influx to reach the threshold level required for L-LTP. In these cases, neuromodulatory inputs that favor voltage-sensitive interactions between these $\mathrm{Ca}^{2+}$ channels, as exemplified in our experiments by the $\beta$-adrenergic inhibition of Kv4.2 channels, would be critical for determining whether synaptic activity at naturally occurring intensities leads to mTOR stimulation and L-LTP. With the ISO-wHFS induction protocol, the role of L-type VGCCs in mTOR activation could be especially prominent, since the contribution of these channels to $\mathrm{Ca}^{2+}$ entry during dendritic spiking is strongly enhanced by $\beta$-adrenergic stimulation (Hoogland and Saggau, 2004).

\section{GSK3 is a tonic suppressor of mTOR and is regulated by the Wnt signaling pathway}

To explore the signaling mechanism that couples Akt to downstream components of the mTOR pathway, we looked for known mTOR regulators that are affected by ISO-wHFS in a VGCC- and NMDAR-dependent manner. Among several candidates, only GSK3, a negative regulator of mTOR, satisfied these criteria. Interestingly, active GSK3 interferes with the induction of LTP (Peineau et al., 2007). Furthermore, GSK3 overexpression disrupts spatial learning, and abnormal regu- 
lation of GSK3 activity has been linked to memory disorders such as Alzheimer's disease (Hernández et al., 2002; Hooper et al., 2008). Our results suggest that the role of GSK3 in gating signal flow through the mTOR pathway, and thus limiting the translational capacity that is required for L-LTP, might help to explain these earlier observations.

A key concept that emerged from our studies is that mTOR activity in the hippocampus, and thus translational capacity, is maintained at a low level by the high basal activity of GSK3, and that this suppression must be overcome for L-LTP to be induced. This hypothesis is supported by the ability of GSK3 inhibitors to strongly activate mTOR in the hippocampus, and to substitute for isoproterenol in enabling weak HFS to induce L-LTP. Remarkably, even though GSK3 is a direct substrate of Akt, strong activation of Akt by isoproterenol alone failed to increase GSK3 phosphorylation, suggesting that Akt and GSK3 are segregated into different subcellular compartments in the absence of synaptic stimulation.

Wnt signaling was required for ISO-wHFS to stimulate mTOR and to induce L-LTP, effects that paralleled the phosphorylation of GSK3 by Akt. It has been proposed that $\mathrm{Ca}^{2+}$ entry through NMDARs mediates the exocytosis of Wnt proteins (Wnts) from the dendrites of hippocampal neurons during strong HFS (Chen et al., 2006). Wnts act as autocrine or paracrine signals at Frizzled-LRP5/6 receptors, disrupting the destruction complex and reducing the access of GSK3 to its substrates within the complex (Inoki et al., 2006; MacDonald et al., 2009). Our data suggest that destruction complex remodeling also renders GSK3 vulnerable to Akt-mediated inhibition, a mechanism that might be widely conserved mechanism since Wnt signaling is required for Akt to phosphorylate GSK3 during ischemic preconditioning in the heart (Vigneron et al., 2011).

If the active Wnt pathway disrupts the destruction complex and prevents GSK3 from interacting with TSC2, is the additional inhibition of GSK3 by Akt a redundant effect? Perhaps not, since phosphorylated GSK3 $\alpha$ might slow the recovery of functional destruction complexes following the termination of the Wnt signal, either by interfering with their reassembly or by becoming incorporated as phosphoproteins into newly assembled complexes. In this way, GSK3 inhibition could prolong the increase in mTOR signaling, allowing the neuron to generate additional translational capacity. Notably, we observed effects of Wnt sequestration by Frizzled-8-Fc and GSK3 inhibition that were more pronounced than those reported in mouse dentate gyrus by Chen et al. (2006). These differences may be attributed to several factors, including the synaptic pathways and species where LTP was investigated, and the stronger LTP induction protocol that was used by Chen et al. (2006).

The regulation of GSK3 following ISO-wHFS was specific to the $\alpha$-isoform, with no effect on the more widely studied $\beta$-isoform. These two isoforms, which share $95 \%$ sequence homology and are virtually identical in their catalytic regions, are expressed at similar levels in the adult rat hippocampus (Woodgett, 1990). Moreover, GSK3 $\alpha$ and GSK3 $\beta$ are interchangeable within the destruction complex, indicating a functional redundancy with respect to Wnt signaling (Asuni et al., 2006; Doble et al., 2007). In contrast to our findings in adult rat hippocampus, Peineau et al. (2007) showed GSK3 $\beta$ to be phosphorylated in juvenile hippocampus following strong HFS (the study did not include measurements of GSK3 $\alpha$ ). We were able to replicate their result, and found additionally that strong HFS robustly phosphorylated the $\alpha$-isoform in juvenile hippocampus, while the same stimulation failed to phosphorylate the $\beta$-isoform in adult hippocampus (data not shown). Thus, the synaptically induced phosphorylation of GSK3 is specific to the $\alpha$-isoform only in adult rat hippocampus, suggesting that as the rat reaches adulthood, GSK3 $\beta$ becomes less accessible to Akt.

\section{An integrated model of mTOR stimulation by synaptic activity}

To explain our findings, we propose a model (Fig. $6 F$ ) in which mTOR activity (and consequently translational capacity) is maintained in a low state under basal conditions by the high resting activity of GSK3 within the destruction complex. Weak HFS by itself produces a modest envelope of depolarization that is mediated entirely by synaptic potentials (not dendritic spikes), since active Kv4.2 channels prevent depolarization from reaching the threshold for opening L-type VGCC or NMDAR channels, and little $\mathrm{Ca}^{2+}$ enters during the stimulus train. $\beta$-Adrenergic stimulation has two important effects: (1) it stimulates Akt (a consequence of increased ERK signaling), and (2) it inhibits Kv4.2 channels, making it possible for weak HFS to recruit L-type VGCCs and NMDARs and increasing dendritic $\mathrm{Ca}^{2+}$ influx. We hypothesize that the increase in intracellular $\mathrm{Ca}^{2+}$ enhances activity in the canonical Wnt pathway, perhaps by inducing the exocytosis of Wnts, which modify destruction complexes within the Wnt-releasing neurons and possibly their neighbors. As a consequence of the changes in the destruction complex, GSK3 becomes accessible to inhibition by Akt, and has reduced ability to inhibit mTOR. The resulting activation of mTOR leads to elevated dendritic translational capacity, facilitating the synthesis of the plasticity-related proteins that are essential for L-LTP.

\section{References}

Asuni AA, Hooper C, Reynolds CH, Lovestone S, Anderton BH, Killick R (2006) GSK3 $\alpha$ exhibits $\beta$-catenin and tau directed kinase activities that are modulated by Wnt. Eur J Neurosci 24:3387-3392.

Brown EJ, Beal PA, Keith CT, Chen J, Shin TB, Schreiber SL (1995) Control of p70 s6 kinase by kinase activity of FRAP in vivo. Nature 377:441-446.

Cai X, Liang CW, Muralidharan S, Kao JP, Tang CM, Thompson SM (2004) Unique roles of SK and Kv4.2 potassium channels in dendritic integration. Neuron 44:351-364.

Cammalleri M, Lütjens R, Berton F, King AR, Simpson C, Francesconi W, Sanna PP (2003) Time-restricted role for dendritic activation of the mTOR-p70S6K pathway in the induction of late-phase long-term potentiation in the CA1. Proc Natl Acad Sci U S A 100:14368-14373.

Chen J, Park CS, Tang SJ (2006) Activity-dependent synaptic Wnt release regulates hippocampal long term potentiation. J Biol Chem 281:11910-11916.

Decker MW, McGaugh JL (1991) The role of interactions between the cholinergic system and other neuromodulatory systems in learning and memory. Synapse 7:151-168.

Doble BW, Patel S, Wood GA, Kockeritz LK, Woodgett JR (2007) Functional redundancy of GSK-3alpha and GSK-3beta in Wnt/beta-catenin signaling shown by using an allelic series of embryonic stem cell lines. Developmental cell 12:957-971.

Frey U, Morris RG (1997) Synaptic tagging and long-term potentiation. Nature 385:533-536.

Frödin M, Jensen CJ, Merienne K, Gammeltoft S (2000) A phosphoserineregulated docking site in the protein kinase RSK2 that recruits and activates PDK1. EMBO J 19:2924-2934.

Gafford GM, Parsons RG, Helmstetter FJ (2011) Consolidation and reconsolidation of contextual fear memory requires mammalian target of rapamycin-dependent translation in the dorsal hippocampus. Neuroscience 182:98-104.

Gelinas JN, Banko JL, Hou L, Sonenberg N, Weeber EJ, Klann E, Nguyen PV (2007) ERK and mTOR signaling couple beta-adrenergic receptors to translation initiation machinery to gate induction of protein synthesisdependent long-term potentiation. J Biol Chem 282:27527-27535.

Hahn-Windgassen A, Nogueira V, Chen CC, Skeen JE, Sonenberg N, Hay N 
(2005) Akt activates the mammalian target of rapamycin by regulating cellular ATP level and AMPK activity. J Biol Chem 280:32081-32089.

Hernández F, Borrell J, Guaza C, Avila J, Lucas JJ (2002) Spatial learning deficit in transgenic mice that conditionally over-express GSK-3beta in the brain but do not form tau filaments. J Neurochem 83:1529-1533.

Hoogland TM, Saggau P (2004) Facilitation of L-type $\mathrm{Ca}^{2+}$ channels in dendritic spines by activation of beta2 adrenergic receptors. J Neurosci 24:8416-8427.

Hooper C, Killick R, Lovestone S (2008) The GSK3 hypothesis of Alzheimer's disease. J Neurochem 104:1433-1439.

Inoki K, Ouyang H, Zhu T, Lindvall C, Wang Y, Zhang X, Yang Q, Bennett C, Harada Y, Stankunas K, Wang CY, He X, MacDougald OA, You M, Williams BO, Guan KL (2006) TSC2 integrates Wnt and energy signals via a coordinated phosphorylation by AMPK and GSK3 to regulate cell growth. Cell 126:955-968.

Kwon G, Marshall CA, Liu H, Pappan KL, Remedi MS, McDaniel ML (2006) Glucose-stimulated DNA synthesis through mammalian target of rapamycin (mTOR) is regulated by KATP channels: effects on cell cycle progression in rodent islets. J Biol Chem 281:3261-3267.

Lenz G, Avruch J (2005) Glutamatergic regulation of the p70S6 kinase in primary mouse neurons. J Biol Chem 280:38121-38124.

Logan CY, Nusse R (2004) The Wnt signaling pathway in development and disease. Annu Rev Cell Dev Biol 20:781-810.

Ma T, Hoeffer CA, Capetillo-Zarate E, Yu F, Wong H, Lin MT, Tampellini D, Klann E, Blitzer RD, Gouras GK (2010) Dysregulation of the mTOR pathway mediates impairment of synaptic plasticity in a mouse model of Alzheimer's disease. PloS One 5:e12845.

MacDonald BT, Tamai K, He X (2009) Wnt/beta-catenin signaling: components, mechanisms, and diseases. Dev Cell 17:9-26.

Manning BD, Cantley LC (2007) AKT/PKB signaling: navigating downstream. Cell 129:1261-1274.

Meyuhas O, Hornstein E (2000) Translational control of TOP mRNAs. In: Translational control of gene expression (Sonenberg N, Hershey JWB, Mathews MB, eds), pp 671-693. Cold Spring Harbor, NY: Cold Spring Harbor Laboratory.

O’Dell TJ, Connor SA, Gelinas JN, Nguyen PV (2010) Viagra for your synapses: enhancement of hippocampal long-term potentiation by activation of beta-adrenergic receptors. Cell Signal 22:728-736.

Peineau S, Taghibiglou C, Bradley C, Wong TP, Liu L, Lu J, Lo E, Wu D, Saule E, Bouschet T, Matthews P, Isaac JT, Bortolotto ZA, Wang YT, Collingridge GL (2007) LTP inhibits LTD in the hippocampus via regulation of GSK3beta. Neuron 53:703-717.

Peterson RT, Beal PA, Comb MJ, Schreiber SL (2000) FKBP12-rapamycinassociated protein (FRAP) autophosphorylates at serine 2481 under translationally repressive conditions. J Biol Chem 275:7416-7423.

Sancak Y, Thoreen CC, Peterson TR, Lindquist RA, Kang SA, Spooner E, Carr
SA, Sabatini DM (2007) PRAS40 is an insulin-regulated inhibitor of the mTORC1 protein kinase. Mol Cell 25:903-915.

Schrader LA, Birnbaum SG, Nadin BM, Ren Y, Bui D, Anderson AE, Sweatt JD (2006) ERK/MAPK regulates the Kv4.2 potassium channel by direct phosphorylation of the pore-forming subunit. Am J Physiol Cell Physiol 290:C852-861.

Srivastava AK, Pandey SK (1998) Potential mechanism(s) involved in the regulation of glycogen synthesis by insulin. Mol Cell Biochem 182: $135-141$.

Stoica L, Zhu PJ, Huang W, Zhou H, Kozma SC, Costa-Mattioli M (2011) Selective pharmacogenetic inhibition of mammalian target of Rapamycin complex I (mTORC1) blocks long-term synaptic plasticity and memory storage. Proc Natl Acad Sci U S A 108:3791-3796.

Tang X, Wang L, Proud CG, Downes CP (2003) Muscarinic receptormediated activation of p70 S6 kinase 1 (S6K1) in 1321N1 astrocytoma cells: permissive role of phosphoinositide 3-kinase. Biochem J 374: 137-143.

Teyler TJ, Cavus I, Coussens C, DiScenna P, Grover L, Lee YP, Little Z (1994) Multideterminant role of calcium in hippocampal synaptic plasticity. Hippocampus 4:623-634.

Tsokas P, Grace EA, Chan P, Ma T, Sealfon SC, Iyengar R, Landau EM, Blitzer RD (2005) Local protein synthesis mediates a rapid increase in dendritic elongation factor $1 \mathrm{~A}$ after induction of late long-term potentiation. J Neurosci 25:5833-5843.

Tsokas P, Ma T, Iyengar R, Landau EM, Blitzer RD (2007) Mitogenactivated protein kinase upregulates the dendritic translation machinery in long-term potentiation by controlling the mammalian target of rapamycin pathway. J Neurosci 27:5885-5894.

Tully K, Bolshakov VY (2010) Emotional enhancement of memory: how norepinephrine enables synaptic plasticity. Mol Brain 3:15.

Vander Haar E, Lee SI, Bandhakavi S, Griffin TJ, Kim DH (2007) Insulin signalling to mTOR mediated by the Akt/PKB substrate PRAS40. Nat Cell Biol 9:316-323.

Vigneron F, Dos Santos P, Lemoine S, Bonnet M, Tariosse L, Couffinhal T, Duplaà C, Jaspard-Vinassa B (2011) GSK-3b at the crossroads in the signalling of heart preconditioning: implication of mTOR and Wnt pathways. Cardiovasc Res 90:49-56.

Wang L, Harris TE, Roth RA, Lawrence JC Jr (2007) PRAS40 regulates mTORC1 kinase activity by functioning as a direct inhibitor of substrate binding. J Biol Chem 282:20036-20044.

Woodgett JR (1990) Molecular cloning and expression of glycogen synthase kinase-3/factor A. EMBO J 9:2431-2438.

Yuan LL, Adams JP, Swank M, Sweatt JD, Johnston D (2002) Protein kinase modulation of dendritic $\mathrm{K}^{+}$channels in hippocampus involves a mitogen-activated protein kinase pathway. J Neurosci 22:4860-4868. 